04

\title{
О выборе параметров высокочастотного индукционного плазмотрона и дисперсного потока испаряемых кварцевых частиц
}

\author{
(C) L. Miao, Ю.М. Гришин \\ Московский государственный технический университет им. Н.Э. Баумана, \\ 105005 Москва, Россия \\ e-mail: miaolongbmstu@gmail.com
}

Поступило в Редакцию 16 ноября 2019 г.

В окончательной редакции 26 ноября 2019 г.

Принято к публикации 27 января 2020 г.

В рамках модели двухфазной бесстолкновительной монодисперсной смеси выполнено численное моделирование процессов нагрева и испарения кварцевых частиц в потоке аргоновой плазмы высокочастотного индукционного плазмотрона. Определены условия осуществления режимов течения с фронтальным вихрем кольцевого типа с полным и частичным проникновением частиц в высокотемпературную зону разряда. Установлены зависимости степени испарения и удельных энергетических затрат на испарение от основных рабочих параметров процесса переработки частиц в первую очередь массового расхода кварцевых частиц, мощности плазмотрона, а также размера и угла ввода частиц в плазменный поток. Даны рекомендации по выбору оптимальных режимных параметров плазмотрона и параметров потока перерабатываемых частиц кварца. Показано, что высокочастотный индукционный плазмотрон мощностью около $5 \mathrm{~kW}$ обеспечивает полное испарение потоков кварцевых частиц с размерами до $50-70 \mu \mathrm{m}$ с расходом до $(8-10) \cdot 10^{-5} \mathrm{~kg} / \mathrm{s}$ при удельных энергетических затратах на уровне $50 \mathrm{MJ} / \mathrm{kg}$.

Ключевые слова: ВЧИ-разряд, режимы течения, степень испарения, оптимальные режимные параметры.

DOI: 10.21883/JTF.2020.07.49438.7-19

\section{Введение}

Необходимость разработки высокоэффективных плазмохимических технологий синтеза углеродных наноструктур [1-3], получения особо чистых веществ [4] и наночастиц [5,6], включающих стадию испарения твердых углеродных, металлических и керамических порошков с применением индукционной плазмы, на протяжении многих лет вызывает значительный интерес к расчетнотеоретическому изучению процессов в высокочастотных индукционных (ВЧИ) плазмотронов [7,8].

В работах [9-13] были применены двумерные модели двухфазной бесстолкновительной моно- и полидисперсной смеси, позволившие установить влияние расходов транспортирующего газа и частиц, теплофизических свойств и размеров частиц, угла ввода частиц в поток, мощности и частоты тока разряда ВЧИ-плазмотрона на эффективность испарения.

В разрабатываемых [14-16] плазменных технологиях получения поликристаллического кремния этап испарения кварцевых частиц в плазменном потоке является особо важным, в известной степени определяющим итоговую эффективность всего процесса. Для обеспечения высокой чистоты получаемого кремния именно на этом этапе необходимо минимизировать загрязнение дисперсного плазменного потока испаряемых кварцевых частиц, а следовательно, проводить переработку кварцевых частиц в ВЧИ-плазмотроне. Между тем к настоящему времени количественная информация об эффективно- сти испарения кварцевых частиц в плазменном потоке ВЧИ-плазмотрона практически отсутствует.

В настоящей работе в рамках модели двухфазной бесстолкновительной монодисперсной смеси выполнено численное моделирование процессов нагрева и испарения кварцевых частиц в потоке аргоновой плазмы ВЧИ-плазмотрона. Главной задачей являлось установление зависимости эффективности испарения от основных рабочих параметров процесса переработки частиц в первую очередь массового расхода кварцевых частиц, мощности ВЧИ-плазмотрона, а также размера и условий ввода частиц в плазменный поток.

\section{Физико-математическая модель}

Исследование проведено для типичной конфигурации (рис. 1) технологического ВЧИ-плазмотрона с трехвитковым индуктором, осевой подачей газов и вертикальным расположением. Размеры основных элементов: $Z_{1}=Z_{2}=50 \mathrm{~mm}, Z_{\text {inf }}=63 \mathrm{~mm}, Z_{\text {inl }}=123 \mathrm{~mm}$, $Z_{3}=400 \mathrm{~mm}, R_{1}=1.7 \mathrm{~mm}, R_{2}=18.8 \mathrm{~mm}, R_{3}=25 \mathrm{~mm}$, $R_{4}=33 \mathrm{~mm}, \quad d_{\text {coil }}=6 \mathrm{~mm}, \quad \delta_{1}=2 \mathrm{~mm}, \quad \delta_{3}=2.2 \mathrm{~mm}$, $\delta_{3}=3.5 \mathrm{~mm}$. В радиальном направлении расчетная область ограничена размером $R_{5}=125 \mathrm{~mm}$. Расходы плазмообразующего и охлаждающего газов равны соответственно $Q_{2}=3.41 / \mathrm{min}$ и $Q_{3}=35.41 / \mathrm{min}$. Транспортирующий газ с расходом $Q_{1}=0.5-51 / \mathrm{min}$, протекающий по центральной трубке (инжектору), обеспечивает подачу кварцевых частиц в зону разряда. Массовый расход дисперсного потока кварцевых частиц $G_{\mathrm{SiO}_{2}}$ варьировался в 


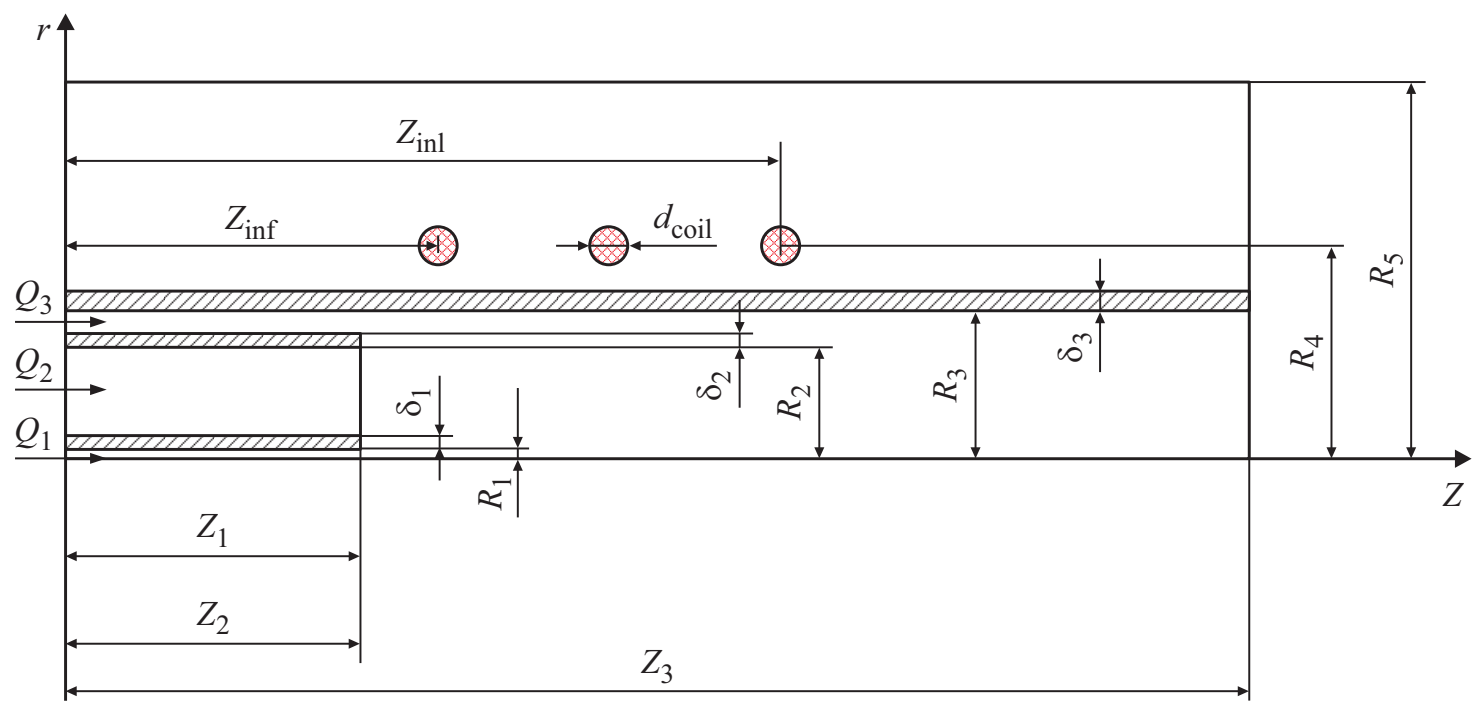

Рис. 1. Схема ВЧИ-плазмотрона: 1 - канал подачи транспортирующего газа и кварцевых частиц, 2 - канал подачи плазмообразующего газа, 3 - периферийный щелевой канал подачи охлаждающего газа.

диапазоне $1 \cdot 10^{-1} G_{\mathrm{Ar}}$ (значение массового расхода всех газов $\left.G_{\mathrm{Ar}}=1 \cdot 10^{-3} \mathrm{~kg} / \mathrm{s}\right)$. Концентрация частиц равномерно распределена на выходном сечении центральной трубки. Амплитуда тока в индукторе $J_{K}$, изменяющегося по синусоидальному закону с частотой $f=3 \mathrm{MHz}$, выбиралась из диапазона значений $150-250$ А.

При формулировке расчетной модели считали, что спиральный индуктор может быть представлен как система трех параллельных колец, при этом плотность тока равномерно распределена по поперечному сечению колец индуктора. Течение газа и потока твердых частиц на входе во все каналы плазмотрона являлось азимутально-симметричным и стационарным. Выполнение этих допущений позволяет рассматривать $[17,18]$ электромагнитные и газодинамические процессы в ВЧИ-плазмотроне как двухмерные в цилиндрической системе координат $(r, z, \theta)$.

Частицы имеют сферическую форму и начальные диаметры $d_{p 0}=30-100 \mu \mathrm{m}$. Как показали оценки [8], в диапазоне значений массового расхода кварцевых частиц $G_{\mathrm{SiO}_{2}}=\left(10^{-3}-2 \cdot 10^{-1}\right) G_{\mathrm{Ar}}$ течение рассматриваемого дисперсного потока может быть отнесено к классу разреженных течений [19], в которых взаимодействием частиц между собой можно пренебречь. При характерном масштабе времени пребывания частицы в потоке $10^{-2} \mathrm{~s}$ температура в кварцевых частицах размером менее $100 \mu \mathrm{m}$ может считаться равномерно распределенной [19]. Исследование дозвукового течения монодисперсных кварцевых потоков в плазме проведено в рамках двухфазной бесстолкновительной дискретнотраекторной модели „particle source in cell““ при наличии нагрева и испарения частиц $[10,20-22]$. При средних значениях числа $\operatorname{Re}_{g}=2 Q_{\mathrm{Ar}} / \pi R_{3} v$, не превышавших $10^{3}$, стационарный плазменный поток в каналах плазмотрона являлся ламинарным [23].
Полная система уравнений включала систему уравнений Максвелла, систему уравнений двухфазной газовой динамики, систему уравнений движения, нагрева и испарения частиц, а также уравнений теплообмена и массообмена между газовой и твердой фазами [11].

Уравнения Максвелла записаны в квазистационарном приближении через вектор-потенциал $\mathbf{A}\left(0,0, A_{\theta}\right)$ :

$$
\nabla \mathbf{A}=-\mu_{0}\left(\mathbf{j}_{\text {voil }}+\mathbf{j}_{\text {ind }}\right),
$$

где $\mathbf{j}_{\text {coil }}$ и $\mathbf{j}_{\text {ind }}$ - векторы плотности тока соответственно в витках катушки индуктора и индукционного тока в плазме, только $\theta$-ые компоненты которых отличны от нуля. Компоненты напряженности электрического $E_{\theta}$ и магнитного $B_{r}, B_{z}$ полей и индукционного тока $j_{i n d}$ вычисляются по формулам

$$
\begin{aligned}
B_{r}=-\frac{\partial A_{\theta}}{\partial z}, \quad B_{z} & =-\frac{1}{r} \frac{\partial}{\partial r}\left(r A_{\theta}\right), \quad E_{\theta}=-i \omega A_{\theta}, \\
j_{\text {ind }} & =\sigma E_{\theta}=-i \sigma \omega A_{\theta},
\end{aligned}
$$

где $\sigma(T)$ - электропроводность плазмы аргона.

Система стационарных уравнений двухфазной газовой динамики (при наличии взаимной диффузии компонентов смеси - аргона и кварцевого пара) с учетом силы взаимодействия с частицами, действия электромагнитных сил, джоулева тепловыделения и излучения [17] записана в виде

$$
\begin{gathered}
\nabla \cdot(\rho \mathbf{u})=-S_{p m}, \\
\nabla \cdot(\rho \mathbf{u} \otimes \mathbf{u})=-\nabla P+\mathbf{F}_{e m}+\nabla(\mu \nabla \mathbf{u})-\mathbf{S}_{p F}, \\
\nabla \cdot\left(\rho \mathbf{u} h-\frac{\lambda}{c_{p}} \nabla h-\rho D \sum h_{i} \nabla Y_{i}\right)=Q_{J o u l e}-Q_{R a d}-S_{p E}, \\
\nabla \cdot\left(\rho \mathbf{u} Y_{\mathrm{SiO}_{2}}-\rho D \operatorname{grad} Y_{\mathrm{SiO}_{2}}\right)=-S_{p m},
\end{gathered}
$$

где $\rho, \mathbf{u}, \mu$ и $\lambda-$ соответственно плотность, вектор скорости, динамическая вязкость и теплопроводность 
газовой смеси, $h=\sum_{i=1}^{2} h_{i} Y_{i}-$ удельная энтальпия смеси газов, $h_{i}$ и $Y_{i}$ - удельная энтальпия и массовая доля $i$-й компоненты $\left(i=1-\mathrm{Ar}, i=2-\right.$ кварцевый пар $\left.\mathrm{SiO}_{2}\right)$, $\sum_{i=1}^{2} Y_{i}=1, D-$ коэффициент диффузии кварцевого пара в аргоне, $Q_{\text {Rad }}=4 \sigma T^{4} / L_{P}(T, P)$ - удельное значение потерь энергии излучения, $L_{P}(T, P)-$ средняя планковская длина пробега кванта света [24], $\mathbf{F}_{\text {em }}$ и $Q_{J o u l e}-$ соответственно удельные (на единицу объема) значения электромагнитной силы и джоулева тепловыделения, которые вычисляются по формулам [17]:

$$
\mathbf{F}_{e m}=\sigma / 2 \operatorname{Re}\left(E_{\theta} B_{z}^{*} \mathbf{r}-E_{\theta} B_{r}^{*} \mathbf{z}\right), \quad Q_{\text {Joule }}=\sigma / 2\left|E_{\theta}\right|^{2} .
$$

Движение и тепломассообмен дисперсной фазы моделируется совокупностью решений для пробных частиц с $k$ точками „старта“ (в нашем случае $k=1,2,3, \ldots 500$ ) в выходном сечении центральной трубки. При этом источниковые члены массы $S_{p m}$, импульса $\mathbf{S}_{p F}$ и тепла $S_{p E}$ между фазами в заданной ячейке $(i, j)$ сетки, используемой для газодинамического расчета, определялись следующим образом $[10,20]$ :

$S_{p m}(i, j)=\sum_{k} n_{(i, j)}^{k} \frac{\Delta m_{p}^{k}}{\tau_{(i, j)}^{k}}, \quad \mathbf{S}_{p F}(i, j)=\sum_{k} n_{(i, j)}^{k} \frac{\Delta\left(m_{p}^{k} \mathbf{u}_{p}^{k}\right)}{\tau_{(i, j)}^{k}}$

$$
S_{p E}(i, j)=\sum_{k} n_{(i, j)}^{k} \frac{Q_{p v}^{k}}{\tau_{(i, j)}^{k}}
$$

где $\Delta m_{p}^{k}$ и $\Delta\left(m_{p}^{k} \mathbf{u}_{p}^{k}\right)$ - изменения массы и импульса в ячейке $(i, j)$ частиц, выходящих из точки $k$, $n_{(i, j)}^{k}=\frac{N_{i} \varphi^{k} \tau_{(i, j)}^{k}}{V_{(i, j)}}$ и $\tau_{(i, j)}^{k}-$ концентрация и время пребывания частиц, выходящих из точки $k$, в ячейке $(i, j)$, $V_{(i, j)}$ - объем ячейки $(i, j), N_{i}=\frac{G_{\mathrm{SiO}_{2}}}{m_{p}}-$ штучный расход частиц из центральной трубки. При равномерном распределении частиц в выходном сечении центральной трубки по 500 точкам „старта“, объемная доля частиц в $k$-й точке $\varphi^{k}=2 \cdot 10^{-3}, Q_{p \nu}^{k}=\int_{0}^{\tau_{(i, j)}^{k}} \alpha_{T}^{k} S_{p}^{k}\left[T_{(i, j)}-T_{p}^{k}\right] d t-$ количество теплоты от газа к частице в ячейке $(i, j)$, $T_{(i, j)}$ - температура газа в ячейке $(i, j), T_{p}^{k}, d_{p}^{k}$ и $S_{p}^{k}=\pi\left(d_{p}^{k}\right)^{2}$ - температура, диаметр и площадь поверхности частиц, $\alpha_{T}^{k}=\left[2+0.6\left(\operatorname{Re}_{p}^{k}\right)^{0.5} \operatorname{Pr}^{0.3}\right] \lambda / d_{p}^{k}-$ коэффициент теплоотдачи между частицами и газом, $\operatorname{Re}_{p}^{k}=\rho d_{p}^{k}\left|\mathbf{u}-\mathbf{u}_{p}^{k}\right| / \mu-$ число Рейнольдса для сферических частиц.

Система уравнений, описывающих движение частицы, стартующей из точки $k$, а также изменение ее темпера- туры и массы вдоль траектории, имеет следующий вид:

$$
\begin{aligned}
& \frac{d\left(m_{p}^{k} \mathbf{u}_{p}^{k}\right)}{d t}=\left(\rho C_{D}^{k} / 8\right) S_{p}^{k}\left|\mathbf{u}-\mathbf{u}_{p}^{k}\right|\left(\mathbf{u}-\mathbf{u}_{p}^{k}\right)+m_{p}^{k} \mathbf{g}, \\
& C_{p m, p} \frac{d\left(m_{p}^{k} T_{p}^{k}\right)}{d t}=\alpha_{T}^{k} S_{p}^{k}\left(T-T_{p}^{k}\right)+\Omega \frac{d m_{p}^{k}}{d t}, \\
& \frac{d m_{p}^{k}}{d t}=\min \left\{\frac{-\alpha_{T}^{k} S_{p}^{k}\left(T-T_{p}^{k}\right)}{\Omega}, \rho k_{c}^{k} S_{p}^{k} \frac{M_{\mathrm{SiO}_{2}}}{\bar{M}}\right. \\
&\left.\quad \times \ln \left(\frac{1-X_{S}^{k}}{1-X_{G}}\right)\right\} .
\end{aligned}
$$

Здесь $m_{p}^{k} \mathbf{g}-$ вектор силы тяжести, $C_{D}^{k}=24 / \operatorname{Re}_{p}^{k}\left[1+0.15\left(R e_{p}^{k}\right)^{0.687}\right] \quad-\quad$ коэффициент сопротивления [25], $C_{p m, p}$ и $\Omega \quad$ - удельная теплоемкость и теплота испарения кварцевых частиц, $k_{c}^{k}=\left[2+0.6\left(\operatorname{Re}_{p}^{k}\right)^{0.5} S c^{0.3}\right] D / d_{p}^{k} \quad$ - коэффициенты массообмена, $M_{\mathrm{SiO}_{2}}$ и $\bar{M}-$ молярная масса кварца и смеси газа, $X_{S}^{k}$ и $X_{G}$ - молярная доля кварцевого пара соответственно на поверхности частиц и в газовой смеси в точке, где находится частица, $X_{S}^{k}$ определяется по величине концентрации насыщенного кварцевого пара (из уравнения Клапейрона-Клаузиуса [21]) при температуре частицы $T_{p}^{k}$.

Граничные условия для уравнений Максвелла задаются следующим образом: на внешних контурах расчетной области при $z=0, z=Z_{3}$ и $r=R_{5}$ величина $A_{\theta}=0$. На оси канала при $r=0$ задается краевое условие симметрии: $\frac{\partial A_{\theta}}{\partial r_{\mid r=0}}=0$. На поверхностях витков индуктора и стенок каналов выполняются условия непрерывности и равенства нормальных к поверхности витков и стенок производных магнитного потенциала в материале стенок, газа или плазмы.

Граничные условия для уравнений двухфазной газовой динамики сформулированы следующим образом: скорости газов на стенках равны нулю; температура внешней поверхности кварцевой стенки $\left(r=R_{3}+\delta_{3}\right)$ равна $300 \mathrm{~K}$; на входах $(z=0)$ температура рабочих газов $T=300 \mathrm{~K}$; транспортирующий, плазмообразующий и охлаждающий газы вводятся по оси в канал ВЧИ-плазмотрона с постоянными скоростями $U_{1}=Q_{1} /\left(\pi R_{1}^{2}\right), \quad U_{2}=Q_{2} /\left[\pi\left(R_{2}^{2}-\left(R_{1}+\delta_{1}\right)^{2}\right]\right.$ и $U_{3}=Q_{3} /\left[\pi\left(R_{3}^{2}-\left(R_{2}+\delta_{2}\right)^{2}\right]\right.$ соответственно; центральная трубка имеет водяное охлаждение и имеет постоянную температуру $T=300 \mathrm{~K}$; на выходе $\left(z=Z_{3}\right)$ давление считается постоянным $P=P_{a t m}=10^{5} \mathrm{~Pa}$.

Для решения системы уравнений движения, энергии и динамики испарения частиц полагали, что вводимые в поток частицы с начальным диаметром $d_{p 0}=30-100 \mu \mathrm{m}$ равномерно распределены по выходному сечению центральной трубки $\left(z=Z_{1}\right)$. Скорость частиц в выходном сечении центральной трубки имела значение, равное средней скорости транспортирующего газа в этом сечении. Температура частиц в выходном 
сечении $z=Z_{1}$ равна $T_{p 0}=300 \mathrm{~K}$. Угол наклона скорости частиц к оси плазмотрона (угол ввода частиц) в выходном сечении центральной трубки $\alpha$ варьировался от 0 до $10^{\circ}$. При нулевом значении $\alpha$ моделировалась ситуация с осевым направлением ввода частиц. Ненулевые величины $\alpha$ соответствовали вариантам с конусным вводом потока частиц в плазму.

При численных расчетах считали, что транспортирующим, плазмообразующим и охлаждающим газами являлся аргон. Теплофизические параметры и электропроводность плазмы определялись в приближении локального термодинамического равновесия по известным данным [25,26].

Теплофизические свойства кварцевых частиц определены по данным [27]. Коэффициент диффузии кварцевых паров в аргоне рассчитан с помощью теории Чепмена-Энскога [28]. Краевые и начальные условия для магнитного потенциала и уравнений газодинамики задавались в соответствии с [17].

Система уравнений решена численно в ПК ANSYS методом конечных объемов. При построении сеток использовалась блочная гексагональная структура HEXA_8.

\section{Результаты расчетов и обсуждение}

Известно [29-31], что при отсутствии подачи твердых частиц в плазменном потоке ВЧИ-плазмотрона в результате действия сжимающих электромагнитных сил индукционных токов при определенных условиях формируется фронтальный вихрь тороидальной формы, центр которого находится приблизительно в сечении, проходящем через плоскость первого витка индуктора. Вниз по потоку этот вихрь ограничен сечением, проходящим через середину (плоскость второго витка) индуктора, где в приосевой области давление имеет максимальное значение. При расходе транспортирующего газа $Q_{1} \leq Q_{\mathrm{cr}}\left(J_{K}\right) \approx 1.7-2.21 / \mathrm{min}$ вихрь (типа Бенара [32]) сомкнут на оси плазмотрона. В данном режиме течения в зоне контакта внутренних поверхностей вихревого тора на оси плазмотрона образуется поток, скорость которого направлена навстречу поступающим в разряд потокам транспортирующего и плазмообразующего газов. В случае расходов транспортирующего газа $Q_{1}$, больших $Q_{\mathrm{cr}}$, образуется вихрь кольцевой формы с осевой полостью, через которую основная часть потока транспортирующего газа течет в осевом направлении непосредственно в высокотемпературную область разряда [32]. Причем важно отметить, что скорости движения газа на внутренних поверхностях вихревого тора и скорости осевого потока противоположны.

Кварцевые частицы могут оказать влияние на структуру вихря и их траектории, начиная со значений массовых расходов $G_{\mathrm{SiO}_{2}} \geq 1 \cdot 10^{-3} G_{\mathrm{Ar}}$. При осевой подаче частиц с расходом $G_{\mathrm{SiO}_{2}} \geq 1 \cdot 10^{-2} G_{\mathrm{Ar}}$ и $Q_{1} \geq 0.8 Q_{\mathrm{cr}}\left(J_{K}\right)$ формирующийся фронтальный вихрь имеет кольцевую форму с осевой полостью, через которую протекает основная часть потоков транспортирующего газа и частиц кварца. При этом траектории движения частиц практически прямые линии, параллельные оси плазмотрона (рис. 2). Здесь отметим, что такой режим может осуществляться и при меньшей величине расхода кварца $G_{\mathrm{SiO}_{2}}=\left(10^{-3}-10^{-2}\right) G_{\mathrm{Ar}}$, но при несколько больших значениях расхода транспортирующего газа $Q_{1} \geq Q_{\mathrm{cr}}\left(J_{K}\right)$. Если $G_{\mathrm{SiO}_{2}} \leq 0.01 G_{\mathrm{Ar}}$, а расход транспортирующего газа $Q_{1} \leq 0.6 Q_{\mathrm{cr}}\left(J_{K}\right) \approx 1-21 / \mathrm{min}$, то реализуются либо бинарный режим, либо кольцевой режим с частичным захватом относительно легких частиц $\left(d_{p 0} \leq 50 \mu \mathrm{m}\right)$ в вихревую зону.

Если ввод частиц из центральной трубки в плазменный поток осуществляется в пределах конуса с углом раскрытия $2 \cdot \alpha$, то при $\alpha \leq 6^{\circ}$ кольцевой вихревой режим с полным проникновением частиц в высокотемпературную зону разряда осуществляется при $Q_{1} \geq Q_{\mathrm{cr}}$ и $G_{\mathrm{SiO}_{2}} \geq 10^{-2} G_{\mathrm{Ar}}$. В данных условиях „веретенообразные“ траектории частиц группируются вокруг оси плазмотрона (рис. 2). Для $\alpha>6-8^{\circ}$ при значениях расходов транспортирующего газа $Q_{1} \leq 0.9 Q_{\mathrm{cr}}\left(J_{K}\right)$ и твердых частиц $\left(G_{\mathrm{SiO}_{2}}=\left(10^{-3}-10^{-1}\right) G_{\mathrm{Ar}}\right)$ имеет место кольцевой режим с частичным захватом частиц в вихревую зону. Подобные режимы, для которых значительная часть частиц из периферийных областей потока может быть выведена из направленного по оси движения (с возможным попаданием на стенки плазмотрона), не являются оптимальными при осуществлении процесса испарения частиц.

При массовом расходе твердых частиц $G_{\mathrm{SiO}_{2}} \geq 10^{-3} G_{\mathrm{Ar}}$ течение дисперсного потока в канале ВЧИ-плазмотрона в режиме с фронтальным кольцевым вихрем характеризуется наличием приосевой области (рис. 2) двухфазного дисперсного потока испаряющихся частиц кварца в газовой смеси аргона и кварцевых паров с более низким уровнем температуры из-за наличия процессов тепломассообмена газа с частицами. Параметры потока в данной области течения наиболее существенно зависят от величины массового расхода кварца $G_{\mathrm{SiO}_{2}}$, т. е. от эффекта ,загрузки“.

Эффективность испарения частиц нужно оценивать по степени испарения на выходе из канала ВЧИ-плазмотрона $\varepsilon_{k}=G_{\mathrm{SiO}_{2}, g}\left(z=Z_{3}\right) / G_{\mathrm{SiO}_{2}}$ и величине удельных затрат энергии на испарения $\eta$, равной отношению мощности разряда (мощности джоулева тепловыделения $\left.P=\int_{V} Q_{\text {Joule }} d V\right)$ к массовому расходу полученного кварцевого пара: $\eta=P /\left[\varepsilon_{k} G_{\mathrm{SiO}_{2}}\right]$. В приведенных формулах $G_{\mathrm{SiO}_{2}, g}\left(z=Z_{3}\right)=2 \pi \int_{0}^{R_{3}} \rho\left(Z_{3}, r\right) Y_{\mathrm{SiO}_{2}}\left(Z_{3}, r\right) U_{z}\left(Z_{3}, r\right) r d r-$ массовый расход пара в сечении с координатой $Z_{3}=400 \mathrm{~mm}$.

Степень испарения частиц на выходе из канала $\varepsilon_{k}$ существенно зависит от массового расхода кварца $G_{\mathrm{SiO}_{2}}$, исходного диаметра частиц $d_{p 0}$ и способа ввода частиц в поток (рис. 3). При увеличении расхода перерабатываемых частиц кварца $\varepsilon_{k}$ снижается. Например, 


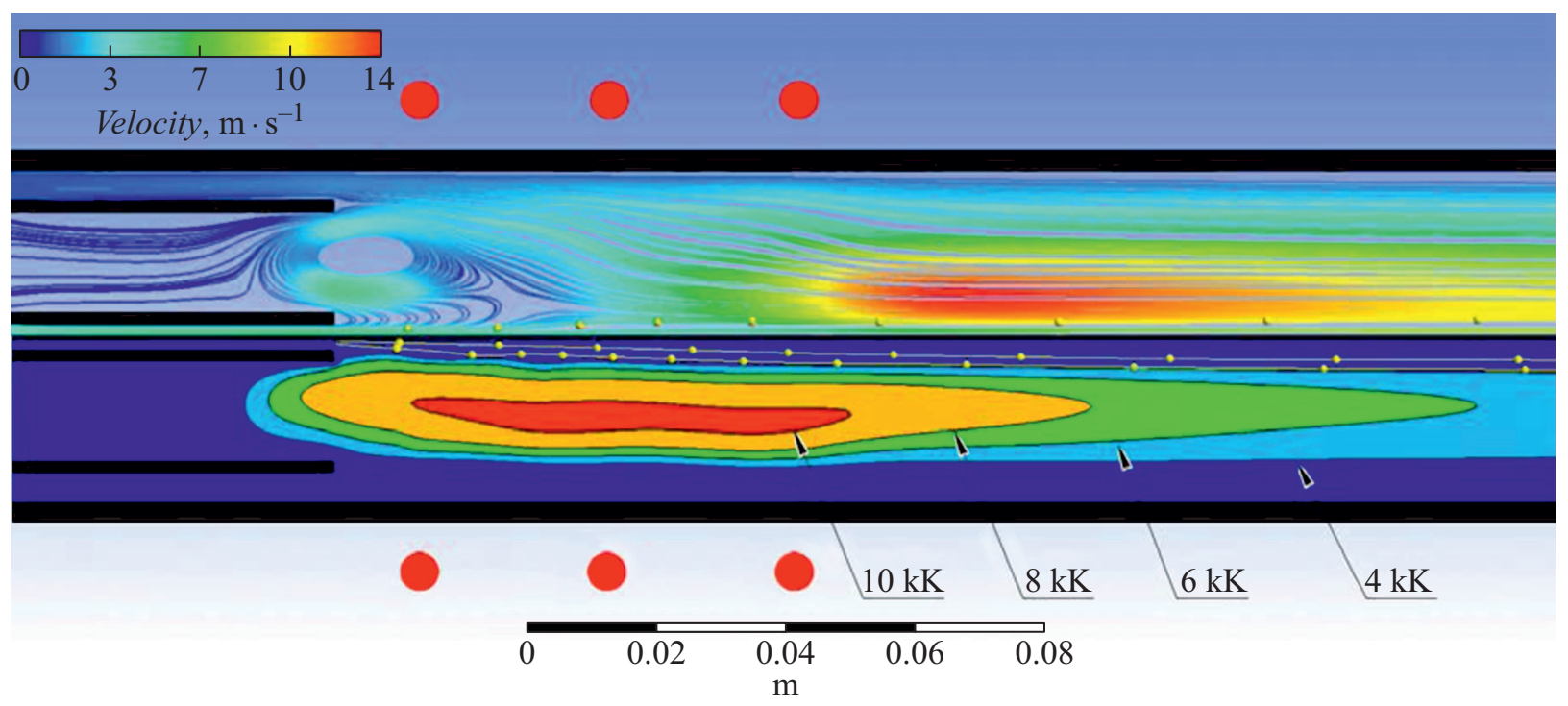

Рис. 2. Линии тока, изолинии температуры и траектории частиц с $d_{p 0}=50 \mu \mathrm{m}$ (линии с точками) при $J_{K}=170 \mathrm{~A}, Q_{1}=2.21 /$ min и различных углах ввода кварцевых частиц: $\alpha=0^{\circ}$ (верхняя полуплоскость); $\alpha=6^{\circ}$ (нижняя полуплоскость).

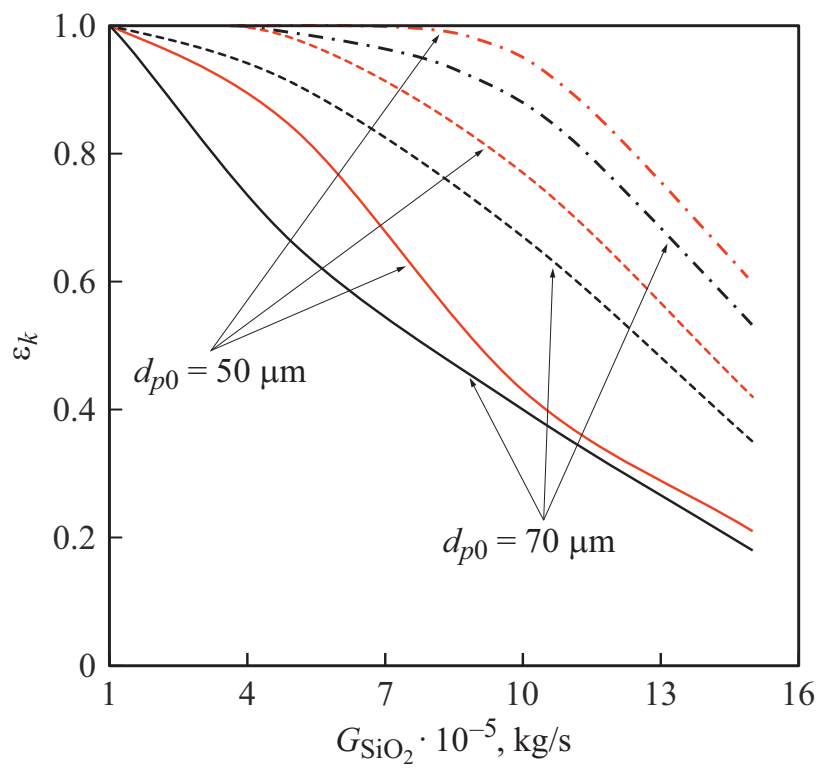

Рис. 3. Зависимость степени испарения кварцевых частиц $\varepsilon_{k}$ от массового расхода частиц кварца $G_{\mathrm{SiO}_{2}}$ : осевой ввод - сплошные линии; конусная инжекция в пределах угла $\alpha=6^{\circ}-$ штриховые линии; кольцевая конусная инжекция под углом $\alpha=6^{\circ}-$ штрихпунктирные линии.

при осевом вводе частиц с диаметром $d_{0}=50 \mu \mathrm{m}$ при $G_{\mathrm{SiO}_{2}} \approx 0.05 G_{\mathrm{Ar}}$ степень испарения около $\varepsilon_{k} \approx 0.8$, а при $G_{\mathrm{SiO}_{2}} \approx 0.1 G_{\mathrm{Ar}}-\varepsilon_{k} \approx 0.4$.

При одном и том же массовом расходе увеличение начального диаметра частиц приводит к снижению степени испарения. Это наиболее заметно при относительно малом расходе частиц $G_{\mathrm{SiO}_{2}}<0.05 G_{\mathrm{Ar}}$. Так, например, при $G_{\mathrm{SiO}_{2}}=0.01 G_{\mathrm{Ar}}$ частицы размером $d_{p 0} \leq 30 \mu \mathrm{m}$ мо- гут полностью испариться к сечению $\mathrm{c}$ координатой $Z_{\kappa} \approx 160 \mathrm{~mm}$, частицы с диаметром $d_{p 0}=50 \mu \mathrm{m}-$ $-Z_{\kappa} \approx 220 \mathrm{~mm}$, а $d_{p 0}=70 \mu \mathrm{m}-Z_{\kappa} \approx 320 \mathrm{~mm}$. Однако при величине массового расхода кварцевых частиц $G_{\mathrm{SiO}_{2}} \geq 0.1 G_{\mathrm{Ar}}$ степень этого влияния ослабевает и, например, значения $\varepsilon_{k}$ для частиц с $d_{p 0}=30-50 \mu \mathrm{m}$ практически одинаковы.

Найдено, что степень испарения относительно слабо (не более $10 \%$ ) зависит от тока (мощности $P$ ) разряда, причем в зависимости от величины $G_{\mathrm{SiO}_{2}}$ - по-разному. При малом расходе $G_{\mathrm{SiO}_{2}} \leq 0.01 G_{\mathrm{Ar}}$ длина участка полного испарения $Z_{T}$ даже несколько увеличивается с ростом $P$ из-за сокращения времени пребывания частицы в зоне испарения. При больших значениях массового расхода $\varepsilon_{k}$ незначительно увеличивается с возрастанием мощности - из-за нагрева приосевой области потока, т. е. из-за снижения эффекта „загрузки“.

Использование способа ввода частиц в поток в пределах конуса с величиной угла раскрытия $2 \cdot \alpha$ значительно влияет на эффективность испарения частиц (рис. 3). При конусной инжекции частиц в поток и расходе кварца степень испарения всегда больше, чем при осевом вводе частиц из-за увеличения радиального размера приосевой области и роста средней температуры газового потока, т.е. в результате снижения влияния эффекта „загрузки“. В диапазоне изменения $2 \cdot \alpha=0-12^{\circ}$, когда захват частиц в вихревую зону практически отсутствует, степень испарения частиц монотонно возрастает (рис. 3) при увеличении угла раскрытия. Например, при переработке частиц диаметром $d_{p 0}=50 \mu \mathrm{m}$ величина $\varepsilon$ может быть в 1.5-2 раза увеличена при изменении угла раскрытия $2 \cdot \alpha$ от 0 до $12^{\circ}$.

Важно обратить внимание (рис. 3), что дополнительное увеличение степени испарения на выходе из канала $\varepsilon$ 
может быть получено за счет использования кольцевого конусного ввода частиц в поток, при котором частицы на выходе из инжектора двигаются только в направлении образующей конуса под углом $\alpha$ к оси плазмотрона. Так, при кольцевой конусной инжекции с углом наклона к оси $6^{\circ}$, ВЧИ-плазмотрон мощностью $5 \mathrm{~kW}\left(J_{K}=170 \mathrm{~A}\right)$ может обеспечить практически полное испарение частиц $\left(d_{p 0}=50 \mu \mathrm{m}\right)$ с максимальным массовым расходом $G_{\mathrm{SiO}_{2}} \approx 0.1 G_{\mathrm{N}}$ (на $20 \%$ больше, чем конусном вводе). При изменении начального размера частиц соответствующим образом изменяется максимальное значение величины расхода перерабатываемых кварцевых частиц: при $d_{p 0}=30 \mu \mathrm{m}-G_{\mathrm{max}} \approx 0.12 G_{\mathrm{Ar}}$, а при $d_{p 0}=70 \mu \mathrm{m}-$ $G_{\text {max }} \approx 0.05 G_{\mathrm{Ar}}$.

Интересно отметить различие в характере влияния тока разряда на $\varepsilon_{k}$ для осевого и конусного ввода частиц при большом $\left(G_{\mathrm{SiO}_{2}} \geq 0.05 G_{\mathrm{Ar}}\right)$ расходе частиц. При осевом вводе частиц увеличение тока приводит к росту $\varepsilon_{k}$. В условиях конусного (и кольцевого конусного) ввода частиц при возрастании тока разряда температура в приосевой области хотя и увеличивается (снижая влияние эффекта „загрузки“ ), но при этом одновременно заметно растет скорость газа и уменьшается время пребывания частицы в газе, так что в результате (в отличие от случая с осевым вводом частиц) степень испарения частиц $\varepsilon_{k}$ даже несколько снижается с возрастанием тока разряда от 170 до $230 \mathrm{~A}$.

Величина затрат $=P /\left[\varepsilon_{k} G_{\mathrm{SiO}_{2}}\right]$ удельных энергохарактеристика эффективности испарения) является немонотонной функцией массового расхода кварцевых частиц $G_{\mathrm{SiO}_{2}}$. При малых значениях $G_{\mathrm{SiO}_{2}}$, когда эффект загрузки несущественен и происходит полное испарение всех частиц, увеличение $G_{\mathrm{SiO}_{2}}$ приводит к росту массового расхода кварцевого пара $G_{\mathrm{SiO}_{2}, g\left(z=Z_{3}\right)}$, а следовательно, к уменьшению $\eta$. При большой величине $G_{\mathrm{SiO}_{2}}$ и существенном влиянии эффекта загрузки рост $G_{\mathrm{SiO}_{2}}$ приводит к уменьшению интенсивности испарения, а следовательно, к снижению $G_{\mathrm{SiO}_{2}, g}\left(z=Z_{3}\right)$ и соответственно к увеличению $\eta$, т.е. величина $\eta$ имеет минимум $\eta_{\min }$ при некотором значении $G_{\mathrm{SiO}_{2}}=G_{\text {opt }}$.

При осевом вводе частиц рассчитанные величины оптимального (с энергетической точки зрения) значения расхода $G_{\text {opt }}$ и $\eta_{\min }$ как функции исходного диаметра частиц $d_{p 0}$ приведены на рис. 4. Видно, что, поскольку с увеличением $d_{p 0}$ величина $\eta_{\min }$ монотонно возрастает, энергетическая эффективность испарения крупных частиц хуже, чем мелких. Следует помнить, что в каждом конкретном случае существует максимальное значение расхода кварцевых частиц $G_{\max }$, при котором имеет место полное испарение всех частиц на выходе канала ВЧИ-плазмотрона $\left(\varepsilon_{k}=1\right)$. Точка пересечения линий $G_{\text {opt }}\left(d_{p 0}\right)$ и $G_{\max }\left(d_{p 0}\right)$ указывает область оптимальных режимных параметров потока испаряемых частиц.

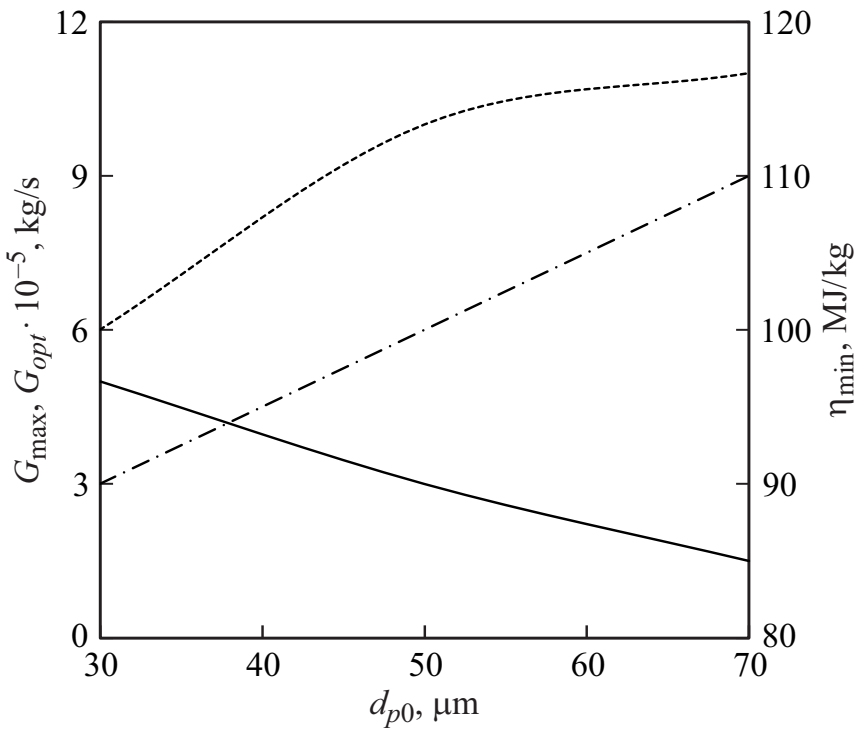

Рис. 4. Зависимости величин максимального испаряемого расход $G_{\max }$ (сплошная линия), оптимального массового расхода $G_{o p t}$ (штриховая линия) и минимальных удельных энергозатрат $\eta_{\min }$ (штрихпунктирная линия) от исходного диаметра частиц $d_{p 0}$ при осевом вводе частиц.

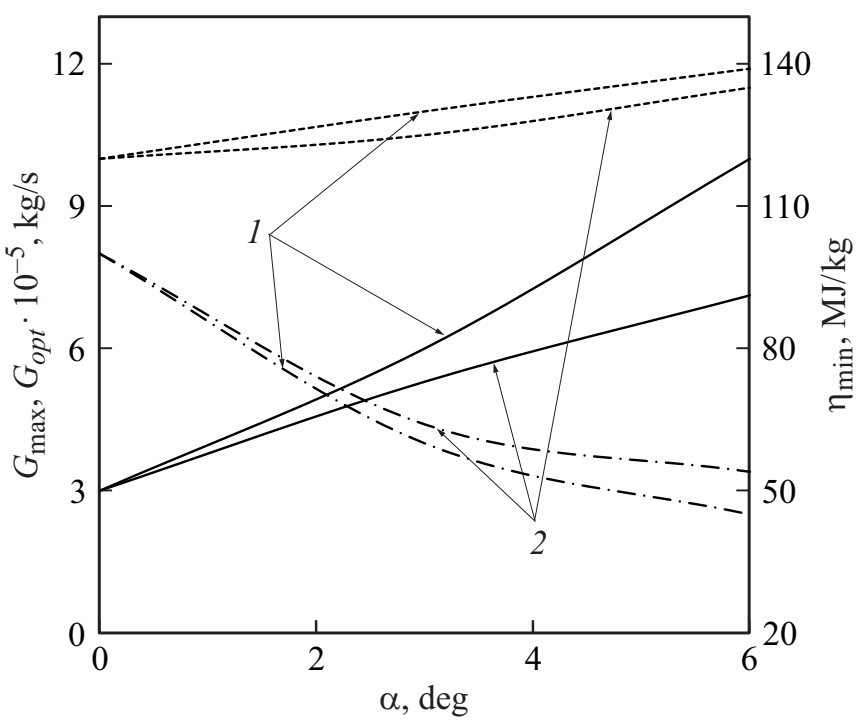

Рис. 5. Зависимости максимального испаряемого расхода $G_{\max }$ (сплошные линии), оптимального массового расхода $G_{o p t}$ (штриховые линии) и минимальных удельных энергозатрат $\eta_{\min }$ (штрихпунктирные линии) от угла ввода частиц при $d_{p 0}=50 \mu \mathrm{m}: 1$ - кольцевой конусный ввод; 2 - конусный ввод.

В частности, как следует из рис. 4, для плазмотрона мощностью $P=5 \mathrm{~kW}$ при осевом вводе частиц оптимально испарять частицы размером около $30 \mu \mathrm{m}$ с расходом $G_{\mathrm{SiO}_{2}}=G_{\max } \approx G_{o p t}=5 \cdot 10^{-5} \mathrm{~kg} / \mathrm{s}$. В этих условиях можно обеспечить полное испарение кварцевых частиц с минимальными энергозатратами $\eta_{\min } \approx 90 \mathrm{MJ} / \mathrm{kg}$. Полное испарение частиц с $d_{p 0}=50 \mu \mathrm{m}$ 
может быть проведено при значительно меньших расходах $G_{\max }=3 \cdot 10^{-5} \mathrm{~kg} / \mathrm{s}$, величина которого почти в 3 paза меньше энергетически оптимального значения $G_{\text {opt }}$ (в этом случае $\eta \approx 220 \mathrm{MJ} / \mathrm{kg}$ ).

Как видно из рис. 5, более низкие удельные энергозатраты по сравнению с осевым вводом частиц имеют ВЧИ-системы как с конусным, так и с кольцевым конусным вводами частиц. Причем увеличение угла ввода частиц до значения $\alpha=6^{\circ}$ (выше которого начинается захват частиц фронтальным вихрем) приводит к монотонному снижению минимальных удельных энергозатрат $\eta_{\min }$. При этом величина $G_{\text {opt }}$ зависит от $\alpha$ относительно слабо. Теоретически достижимая величина удельных энергозатрат при испарении частиц с $d_{p 0}=50 \mu \mathrm{m}$ находится на уровне $\eta=45-55 \mathrm{MJ} / \mathrm{kg}$ для систем ввода частиц с $\alpha=6^{\circ}$. Следует отметить, что, хотя с ростом $\alpha$ увеличивается максимальное значение расхода перерабатываемых кварцевых частиц $G_{\max }$, однако при конусном вводе частиц его величина в 1.5 меньше $G_{o p t}$. Этот результат свидетельствует о том, что оптимальный режим испарения можно будет осуществить только для частиц с несколько меньшими исходными размерами $d_{p 0}=30-40 \mu \mathrm{m}$.

Применение кольцевого конусного ввода частиц позволяет не только улучшить энергетический показатель эффектиности испарения $\eta$ по сравнению с конусным вводом частиц с $d_{p 0}=50 \mu \mathrm{m}$, но и практически приблизить (при $\alpha=6^{\circ}$ ) максимальное значение расхода перерабатываемых кварцевых частиц $G_{\max }=1 \cdot 10^{-4} \mathrm{~kg} / \mathrm{s} \mathrm{\kappa}$ оптимальному $G_{\text {opt }}=1.2 \cdot 10^{-4} \mathrm{~kg} / \mathrm{s}$ и тем самым реализовать режим испарения с минимальными энергозатратами.

\section{Заключение}

Необходимым условием, обеспечивающим осуществление режима течения дисперсной смеси с фронтальным вихрем кольцевой формы, через осевую полость которого протекает основная часть потоков транспортирующего газа и частиц кварца (без захвата их в вихревое движение), является превышение значения расхода транспортирующего газа $Q_{1}$ некоторой критической величины $Q_{\mathrm{cr}}\left(J_{K}\right)$, значение которого в зависимости от тока разряда находится в диапазоне 1.7-2.21/min при расходе кварцевых частиц (с размерами $\left.d_{p 0} \geq 30 \mu \mathrm{m}\right)-$ $G_{\mathrm{SiO}_{2}} \geq 10^{-3} G_{\mathrm{Ar}}$. Реализация режима с полным проникновением частиц в высокотемпературную зону разряда имеет место как при осевом вводе частиц, так и при вводе частиц из центральной трубки в плазменный поток под углом к оси плазмотрона в пределах конуса с углом раскрытия $2 \cdot \alpha \leq 12^{\circ}$.

Степень испарения частиц на выходе из канала $\varepsilon_{k}$ существенно зависит от массового расхода кварца $G_{\mathrm{SiO}_{2}}$ и диаметра частиц $d_{p 0}$. При увеличении расхода перерабатываемых частиц кварц $\varepsilon_{k}$ снижается. Например, для частиц с диаметром $d_{p 0}=50 \mu \mathrm{m}$ при $G_{\mathrm{SiO}_{2}} \approx 0.05 G_{\mathrm{Ar}}$ эффективность испарения около $\varepsilon_{k}=0.8$, а при $G_{\mathrm{SiO}_{2}} \approx 0.1 G_{\mathrm{Ar}}-\varepsilon_{k} \approx 0.4$. Степень испарения частиц на выходе из канала $\varepsilon_{k}$ при осевом вводе частиц незначительно увеличивается с возрастанием тока (мощности) разряда от 170 до 230 А, а при конусном вводе частиц даже несколько снижается. Поэтому следует считать, что оптимальные значения амплитуды тока ВЧИ-разряда составляют величину около 170 А. Применение конусной инжекции частиц с углом раскрытия $2 \cdot \alpha \leq 12^{\circ}$ при прочих равных условиях повышает степень испарения частиц по сравнению с осевым вводом.

Выбор оптимальных режимных параметров плазменной ВЧИ-системы переработки кварцевых частиц должен осуществляться из условия полного испарения частиц при минимальных энергозатратах, т.е. при выполнении равенств $G_{\mathrm{SiO}_{2}} \approx G_{\max }\left(d_{p 0}, \alpha\right) \approx G_{\text {opt }}\left(d_{p 0}, \alpha\right)$. Максимальная эффективность достигается при применении кольцевой конусной инжекции с $\alpha=6^{\circ}$. Для ВЧИ-плазмотрона мощностью $5 \mathrm{~kW}$ при кольцевой конусной инжекции кварцевых частиц с $d_{p 0}=40-50 \mu \mathrm{m}$ величина полностью испаряемого расхода $G_{\max }=$ $=0.95 \cdot 10^{-4} \mathrm{~kg} / \mathrm{s}$ близка к оптимальной $G_{\text {opt }}=$ $=(1-1.2) \cdot 10^{-4} \mathrm{~kg} / \mathrm{s}$, а следовательно, минимальны удельные энергетические затраты.

\section{Конфликт интересов}

Авторы заявляют, что у них нет конфликта интересов.

\section{Список литературы}

[1] Залогин Г.Н., Красильников А.В., Рудин Н.Ф., Попов М.Ю., Кульницкий Б.А., Кириченко А.Н. // ЖТФ. 2015. T. 85. Вып. 5. C. 100-105. [Zalogin G.N., Krasil'nikov A.V., Rudin N.F., Popovb M.Yu., Kul'nitskii B.A., Kirichenko A.N. // Tech. Phys. 2015. Vol. 60. N 5. P. 730-735.] DOI: $10.1134 / \mathrm{S} 106378421505028 \mathrm{X}$

[2] Власов В. И., Залогин Г.Н., Кусов А.Л. // ЖТФ. 2007. Т. 77. Вып. 1. C. 30-37. [Vlasov V.I., Zalogin G.N., Kusov A.L. // Tech. Phys. 2007. Vol. 52. N 1. P. 27-34.] DOI: $10.1134 / \mathrm{S} 1063784207010057$

[3] Анчуков К.Е., Залогин Г.Н., Красильников А.В., Попов М.Ю., Кульницкий Б.А. // Письма в ЖТФ. 2015. T. 41. Вып. 21. С. 30-37. [Anchukov K.E., Zalogin G.N., Krasil'nikov A.V., Popov M.Yu., Kul'nitskii B.A. // Tech. Phys. Lett. 2015. Vol. 41. N 11. P. 1038-1040.] DOI: $10.1134 / \mathrm{S} 1063785015110024$

[4] Гришин Ю.М., Козлов Н.П., Скрябин А.С. // ТВТ. 2012. T. 50. Вып. 4. С. 491-495. [Grishin Yu.M., Kozlov N.P., Skryabin A.S. // High Temp. 2012. Vol. 50. N 4. P. 459-463.] DOI: $10.1134 / \mathrm{S} 0018151 \mathrm{X} 12040086$

[5] Colombo V., Ghedini E., Gherardi M., Sanibondi P. // Plasma Sources Sci. T. 2013. Vol. 22. N 3. P. 035010 (11p). DOI: $10.1088 / 0963-0252 / 22 / 3 / 035010$

[6] Mendoza Gonzalez N.Y., Morsli M.El., Proulx P. // J. Therm. Spray Technol. 2008. Vol. 17. N 4. P. 533-550. DOI: $10.1007 / \mathrm{s} 11666-008-9209-\mathrm{x}$

[7] Гришин Ю.М., Мяо Л. // Прикладная физика. 2017. Вып. 3. C. $31-36$. 
[8] Grishin Yu.M., Miao L. // J. Physics: Conf. Series. 2017. Vol. 830. P. 012069 (6p). DOI: $10.1088 / 1742-6596 / 830 / 1 / 012069$

[9] Aghaei M., Bogaerts A. // J. Anal. At. Spectrom. 2016. Vol. 31. N 3. P. 631-641. DOI: $10.1039 / \mathrm{c} 5 \mathrm{ja}$ 000162e

[10] Proulx P., Mostaghimi J., Boulos M.I. // Int. J. Heat Mass Transfer. 1985. Vol. 28. N 7. P.1327-1336.

[11] Colombo V., Ghedini E., Sanibondi P. // Plasma Sources Sci. T. 2010. Vol. 19. N 6. P. 065024 (13p). DOI: $10.1088 / 0963-0252 / 19 / 6 / 065024$

[12] Bernardi D., Colombo V., Ghedini E., Mentrelli A., Trombetti T. // Eur. Phys. J. D. 2004. Vol. 28. N 3. P. 423-433. DOI: $10.1140 /$ epjd/e2004-00012-8

[13] Shan Y.G. // IEEE T. Plasma Sci. 2009. Vol. 37. N 9. P. 1747-1753. DOI: 10.1109/TPS.2009.2028141

[14] Гришин Ю.М., Козлов Н.П., Скрябин А.С. // TBT. 2016. Т. 54. Вып. 5. С. 655-662. DOI: $\quad 10.7868 / \mathrm{S} 0040364416040086$ Grishin Yu.M., Kozlov N.P., Skryabin A.S. // High Temp. 2016. Vol. 54. N 5. P. 619-626. DOI: 10.1134/S0018151X16040088]

[15] Ma W.H., Ogura M., Kobayashi T., Takahashi H. // Sol. Energy Mater. Sol. Cells. 2004. Vol. 81. N 4. P. 477-483. DOI: $10.1016 /$ j.solmat.2003.12.001

[16] Mexmain J.M., Morvan D., Bourdin E., Amouroux J., Fauchais P. Plasma Chem. and Plasma Proces. 1983. Vol. 3. N 4. P. $393-420$.

[17] Miao L., Grishin Yu.M. // Plasma Sources Sci. T. 2018. Vol. 27. N 11. P. 115008 (11p). DOI: 10.1088/1361-6595/aae8f2

[18] Васильевский С.А., Колесников А.Ф. // Изв. РАН. МЖГ. 2000. Вып. 5. С. 164-173. [Vasil'evskii S.A., Kolesnikov A.F. // Fluid Dynamics. 2000. N 5. P. 769-777.]

[19] Дресвин С.В., Михальков С.М. // ТВТ. 1992. Т. 30. Вып. 1. C. 25-35. [Dresvin S.V., Mikhalkov S.M. // High Temp. 1992. Vol. 30. N 1. P. 21-30.]

[20] Crowe C.T., Sharma M.P., Stock D.E. // J. Fluids Eng. 1977. Vol. 99. N 2. P. $325-332$. DOI: $10.1115 / 1.3448756$

[21] Нигматулин Р.И. Динамика многофазных сред Ч. І. М.: Наука, 1987. 464 с.

[22] Волков К.Н., Емельянов В.Н. Течения газа с частицами. М.: Физматлит, 2008. 600 c.

[23] Boulos M.I. // Pure\&Appl. Chem. 1985. Vol. 57. N 9. P. $1321-1352$.

[24] Кацнельсон С.С., Ковальскал Г.А. Теплофизические и оптические свойства аргоновой плазмы. Новосибирск: Наука, 1985. 147 c.

[25] Boulos M.I., Fauchais P., Pfender E. Thermal Plasmas: Fundamentals and Applications. Vol. 1. NY.: Plenum Press, 1994. $452 \mathrm{p}$.

[26] Дресвин С.В. Основы теории и расчета высокочастотных плазмотронов. Л.: Энергоатомиздат, 1991. 312 с.

[27] Бабушкина А.П., Братковский А.М. и др. Физические величины: Справочник. М: Энергоатомиздат, 1991. 1232 с.

[28] Bird R.B., Stewart W.E., Lightfoot E.N. Transport Phenomena, Second Edition. NY.: John Wiley \& Sons, Inc., 2006. 905 p.

[29] Chase J.D. // J. Appl. Phys. 1969. Vol. 40. N 1. P. 318-325. DOI: $10.1063 / 1.1657052$

[30] Utyuzhnikov S.V., Konyukhov A.V., Rudenko D.V., Vasil'evskii S.A., Kolesnikov A.F., Chazot O. // AIAA J. 2004. Vol. 42. N 9. P. 1871-1877. DOI: 10.2514/1.1195

[31] Мяо Л., Гришин Ю.М. // Физика плазмы. 2018. Т. 44. Вып. 11. C. 881-887. DOI: 10.1134/S0367292118110070 [Miao L., Grishin Yu.M. // Plasma Phys. Rep. 2018. Vol. 44. N 11. P. 1019-1025. DOI: 10.1134/S1063780X18110077]

[32] Miao L., Grishin Yu.M. // Int. J. Heat Mass Transfer. 2019. Vol. 144. P. 118671 (12p)

DOI: 10.1016/j.ijheatmasstransfer.2019.118671 